Article

\title{
An Active Self-Driven Piezoelectric Sensor Enabling Real-Time Respiration Monitoring
}

\author{
Ahmed Rasheed 1,2®, Emad Iranmanesh ${ }^{1}$, Weiwei Li ${ }^{1,2}$, Yangbing Xu ${ }^{1,2}$, Qi Zhou ${ }^{1,2}$, Hai Ou ${ }^{1}$ \\ and Kai Wang 1,2,*(D) \\ 1 Guangdong Province Key Laboratory of Display Material and Technology, State Key Laboratory of \\ Optoelectronic Materials and Technologies, School of Electronics and Information Technology, Sun Yat-sen \\ University, No. 132 East Waihuan Road, Guangzhou 510006, China \\ 2 Sun Yat-sen University Shunde Research Institute, No. 9 Eastern Nanguo Road, Shunde District, \\ Foshan 523800, China \\ * Correspondence: wangkai23@mail.sysu.edu.cn
}

Received: 13 June 2019; Accepted: 18 July 2019; Published: 23 July 2019

\begin{abstract}
In this work, we report an active respiration monitoring sensor based on a piezoelectrictransducer-gated thin-film transistor (PTGTFT) aiming to measure respiration-induced dynamic force in real time with high sensitivity and robustness. It differs from passive piezoelectric sensors in that the piezoelectric transducer signal is rectified and amplified by the PTGTFT. Thus, a detailed and easy-to-analyze respiration rhythm waveform can be collected with a sufficient time resolution. The respiration rate, three phases of respiration cycle, as well as phase patterns can be further extracted for prognosis and caution of potential apnea and other respiratory abnormalities, making the PTGTFT a great promise for application in long-term real-time respiration monitoring.
\end{abstract}

Keywords: thin-film transistor; piezoelectric transducer; active sensor; self-driven sensor; respiration monitoring

\section{Introduction}

Respiratory diseases are the second leading $(\sim 6.5 \%)$ cause of mortality globally [1]. Fortunately, they are preventable if a reliable and accurate monitoring method is implemented. Therefore, real-time monitoring of respiratory rhythm that provides ample information about breathing trend becomes critical [2-5]. Moreover, study of respiratory patterns and phases helps diagnose pulmonary illness, such as apnea [6], asthma [7], and chronic obstructive pulmonary diseases [8]. Conventionally, electrocardiogram (ECG) $[9,10]$ and transthoracic impedance plethysmography (TI-PPG) [11-13] are widely used to perform non-invasive monitoring of respiration rate. ECG monitors electrical stimulus response of lungs from multiple leads and TI-PPG monitors small change in tissue volumes upon respiration. Both require the examined body to be immobile during examination for accurate and reliable measurements. Therefore, such commercially-available devices are not well-suited for wearable and real-time monitoring in daily activities. In addition, they are bulky and consume a lot of power, which is inconvenient for long-term operation.

Other than ECG and PPG, various new wearable respiration rate sensors have also been investigated. Supplementary Figure S1 summarizes these sensors in terms of sensing mechanisms and sensing materials. Regarding sensing mechanisms, transduction of mechanical, electrical, thermal, and vaporous stimuli to electrical signals are typical. Among them, direct detection of air-movement upon respiration process using new-fashioned sensing materials has become popular since it is not affected by motion artifacts [14-16]. For example, a paper-based humidity sensor, adjustable inside a wearing mask, has been used for respiration rate monitoring through humidity measurement of 
conductive paper [14]. Impedance variation with water absorption of silica nanoparticles thin-film on flexible substrate was measured to extract respiration rate [16]. However, such sensors are very difficult to wear and are also affected by environmental humidity, thus giving unreliable data. Similarly, flexible and wearable pressure sensors can directly measure pressure change during the respiration process and they exhibit good features, such as being light and portable [17-19]. These sensors can be based on piezoelectric effect $[20,21]$, capacitance change [22,23], and thermoelectric effect $[24,25]$ in response of pressure change. However, they are passive devices with limited sensitivity and dynamic range. More recently, a hollow micro-structured pressure sensor offering a wide dynamic range has been reported [26]. However, it is non-contact based, limited to static pressure measurements, and its response time is problematic due to deformation dependence on ethylene-vinyl acetate (EVA) film structure. Respiration rate sensors based on triboelectric and pyroelectric effect with high sensitivity were very attractive [24,27]. However, their super high sensitivities lead to reliability issues arising from mechanical, motion, and electrostatic shocking. The long-term usage can also cause material/structural fatigue as a result of frequent friction.

In this work, we propose an active self-driven wearable piezoelectric sensor intended for real-time respiration monitoring. The device architecture will be presented along with the analytical modeling of sensitivity. The respiration rates and rhythm at multiple body peripheral during various physical activities will be obtained and the transient response of the sensor by detecting all respiratory patterns and phases will be also given. The feasibility of using this sensor for real-time respiration monitoring has been discussed particularly for mobile health care. Compared with other wearable respiration sensors, this proposed sensor is very competitive in terms of sensitivity, signal-to-noise ratio (SNR), dynamic range, operating voltage, power consumption, response time, and device fabrication process as listed in Table 1.

Table 1. Summary of this research compared with the prior arts in terms of sensitivity, signal-to-noise ratio (SNR), dynamic range, driving voltage, response time, and power consumption.

\begin{tabular}{|c|c|c|c|c|c|c|c|}
\hline Sensor System & $\begin{array}{l}\text { Operating } \\
\text { Voltage } \\
\text { (V) }\end{array}$ & $\begin{array}{c}\text { Power } \\
\text { Consumption } \\
\text { (W) }\end{array}$ & $\begin{array}{l}\text { Response } \\
\text { Time (sec) }\end{array}$ & $\begin{array}{l}\text { Dynamic } \\
\text { Range }\end{array}$ & $\begin{array}{l}\text { Sensitivity } \\
(@ 0.5 \mathrm{~N})\end{array}$ & SNR (dB) & $\begin{array}{l}\text { Device } \\
\text { Process }\end{array}$ \\
\hline $\begin{array}{l}\text { Triboelectric } \\
\text { Based Sensing } \\
\text { Method [27] }\end{array}$ & $\begin{array}{l}\text { Self-Driven } \\
\quad(\sim 0 \mathrm{~V})\end{array}$ & $<1 \mathrm{~m}$ & $\sim 100 \mathrm{~m}$ & $\begin{array}{c}\sim 0.2 \mathrm{~N} \text { to } \\
10 \mathrm{~N}\end{array}$ & $\sim 600 \mathrm{mV}$ & 45 & $\begin{array}{l}\text { Low cost and } \\
\text { simple }\end{array}$ \\
\hline $\begin{array}{l}\text { Humidity Based } \\
\text { Measurement [16] }\end{array}$ & $>5 \mathrm{~V}$ & $\sim 20 \mathrm{~m}$ & $\sim 700 \mathrm{~m}$ & - & Low & - & $\begin{array}{l}\text { Low cost and } \\
\text { complex }\end{array}$ \\
\hline $\begin{array}{l}\text { Optical Based } \\
\text { Sensing Method } \\
{[12,13]}\end{array}$ & $>3 \mathrm{~V}$ & $\sim 60 \mathrm{~m}$ & $>1000 \mathrm{~m}$ & - & Low & Low & $\begin{array}{l}\text { Medium cost } \\
\text { and complex }\end{array}$ \\
\hline This Work & $\begin{array}{l}\text { Self-Driven } \\
\quad(\sim 0 \mathrm{~V})\end{array}$ & $\sim 600 \mu$ & $50 \mathrm{~m}$ & $>50 \mathrm{mN}$ & $\sim 800 \mathrm{mV}$ & $>20$ & $\begin{array}{l}\text { Low cost and } \\
\text { simple }\end{array}$ \\
\hline
\end{tabular}

\section{Methods}

\subsection{Sensor Architecture}

The cross-sectional schematic diagram of the proposed sensor is illustrated in Figure 1a. Different from a passive piezoelectric transducer with simply a sandwich structure, an active sensor is architected by a piezoelectric transducer and a dual-gate thin-film transistor (DG-TFT) forming a piezoelectric-transducer-gated TFT (PTGTFT). The sensor is capable of not only detecting a dynamic force signal but also rectifying it into a direct current (DC) signal and further amplifying it. The equivalent circuit diagram for respiration monitoring system based on the PTGTFT is shown in Figure $1 \mathrm{~b}$, where shortening bottom gate (BG), top gate (TG), and drain (D) terminals of the PTGTFT makes a diode-mode connection for signal rectification. Here, the DG-TFT is used instead of single-gate TFT for the sensitivity enhancement and better interfacing with the readout electronics. In this architecture, no external power source is needed and all the biasing voltages for the sensor originate 
from the PVDF transducer, making it self-driven with zero power consumption. The output DC current signal is further handled by an AFE consisting of a low-noise current amplifier and low-pass RC filter before entering the data acquisition module (DAM), which includes an ultra-low power microcontroller unit (MCU) that houses a built-in 12-digit analog-to-digital converter (ADC), an I/O interface, and a LCD panel. Figure 1c shows a photo of the proposed respiration monitoring sensor system.

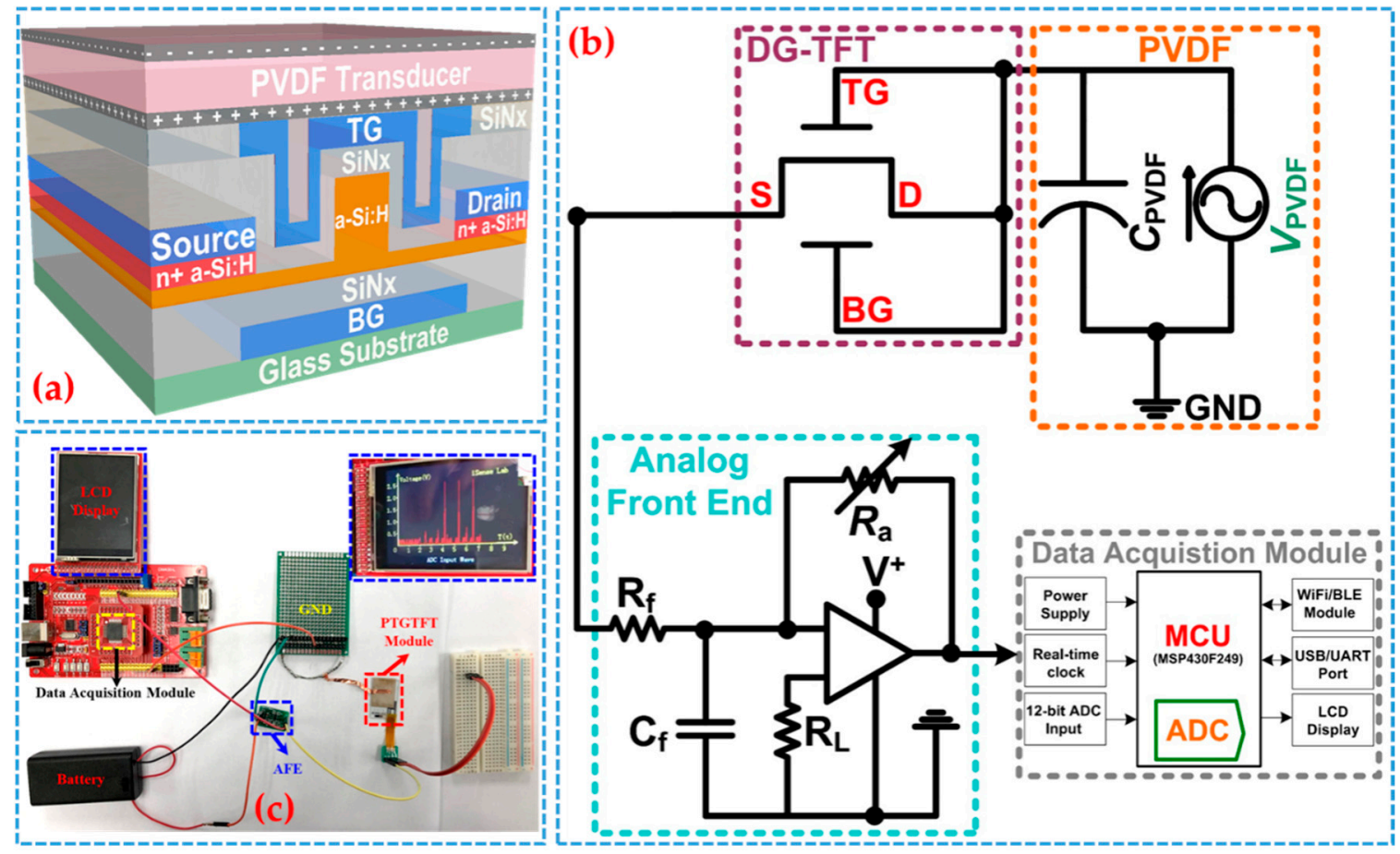

Figure 1. (a) Cross-sectional schematic illustration of a piezoelectric-transducer-gated thin-film transistor (PTGTFT) where a PVDF transducer is connected to a FIN-shaped a-Si:H piezoelectric transducer and a dual-gate thin-film transistor (DG-TFT); (b) equivalent circuit diagram of respiration monitoring sensor system composed of a PTGTFT, low-power analog front end (AFE) and conventional data acquisition module (MSP430F149); and (c) photo of the experimental setup for measuring the respiration rhythm signal by the proposed sensor system.

\subsection{Analytical Modeling}

In the self-driven mode, the top gate voltage $\left(V_{T G}\right)$ is equal to the drain-source voltage $\left(V_{D S}\right)$ and the bottom gate voltage $\left(V_{B G}\right)$, and is given by the piezoelectric-induced charges $\left(Q_{P V D F}\right)$ over the total capacitance, $C_{\text {Total }}$ :

$$
V_{T G}=V_{B G}=V_{D S}=\frac{Q_{P V D F}}{C_{T o t a l}}=\frac{Q_{P V D F}}{\left(C_{P V D F}+C_{T G}+C_{B G}\right)}
$$

where $C_{P V D F}$ : Capacitance of the PVDF transducer, $C_{T G}$ : Top gate capacitance and $C_{B G}$ : Bottom gate capacitance in the DG-TFT. Threshold voltage $\left(V_{T}\right)$ of the DG-TFT is dependent with $V_{T G}$ as follows:

$$
V_{T}=V_{T_{O}}-\gamma \cdot V_{T G}
$$

where $V_{T o}$ is the original threshold voltage of the DG-TFT with applying zero top-gate bias and $\gamma$ indicates the variation in the $V_{T}$ with the $V_{T G}$. The working regime of the PTGTFT depends on its force stimuli. In our previous studies [28-30], a similar device working in the deep-subthreshold regime is used to detect very weak dynamic forces from the heart beating for heart rate monitoring. The respiratory force is generally strong and thus the PVDF transducer generates voltages greater than $V_{T}$ 
(normally smaller than $1 \mathrm{~V}$ ) while $V_{D S}>V_{B G}-V_{T}$ is readily satisfied. As a result, the PTGTFT likely works in the saturation region, giving the output current $\left(I_{D S}\right)$ as below:

$$
I_{D S}=\frac{1}{2} \mu_{F E} \cdot C_{B G} \cdot \frac{W}{L} \cdot\left[\left(V_{B G}-V_{T}\right)\right]^{2}
$$

where $\mu_{F E}$ : Field-effect mobility of the PTGTFT; W: Channel width of the PTGTFT, and L: Channel length of the PTGTFT. Substituting Equations (1) and (2) in Equation (3) gives:

$$
\sqrt{I_{D S}}=\sqrt{\frac{1}{2} \mu_{F E} \cdot C_{B G} \cdot \frac{W}{L}} \cdot\left[\left(\frac{Q_{P V D F}}{\left(C_{P V D F}+C_{T G}+C_{B G}\right)}\right)(1+\gamma)-V_{T_{O}}\right]
$$

The sensitivity, $S$, in the saturation region can be defined as the ratio of the change of the square root of output current, $d \sqrt{I_{D S}}$, to the change of the generated charges by the respiration action, $d Q_{P V D F}$, as follows:

$$
S=\frac{d \sqrt{I_{D S}}}{d Q_{P V D F}}=\sqrt{\frac{1}{2} \mu_{F E} \cdot C_{B G} \cdot \frac{W}{L}} \cdot\left[\left(\frac{(1+\gamma)}{\left(C_{P V D F}+C_{T G}+C_{B G}\right)}\right)\right]
$$

As can be seen, the sensitivity is attributed to the material, design, and process parameters of $\mu_{\mathrm{FE}}$, channel width and length $(\mathrm{W} / \mathrm{L})$ ratio, $\gamma, C_{\text {Total }}$, and $C_{B G}$, among which $\gamma$ is a key parameter dictating how the transducer interacts and couples with the TFT. Our previous study has already found that the value of $\gamma$ can be doubled by using a 3-D FIN-shaped TFT structure as opposed to a regular planar structure [30]. Hence, in this research, we designed and fabricated the PTGTFT with a 3-D FIN-shaped structure.

\subsection{Sensor Fabrication}

The fabrication process included preparation of the 3-D FIN-shaped DG-TFT based on amorphous silicon (a-Si:H) thin-film semiconductor material and processes [30] and the PVDF transducer that was formed by a sandwich structure of silver coated PVDF film (thickness: $52 \mu \mathrm{m}$; supplier: Measurement Specialties Inc., Hampton, VA, USA), i.e., Ag/PVDF/Ag. The process flow of fabricating 3-D FIN-shaped DG-TFT consisted of six photolithographic masks and was based on top-down approach of mainstream a-Si:H TFT process as shown in Supplementary Figure S2. Mask \#1: A Chrome (Cr) metal layer of 100-nm thickness was deposited on glass substrate (Corning 1737) by direct current (DC) sputtering at room temperature followed by wet-etching to pattern photolithography of bottom gate. Mask \#2: A 300-nm-thick $\mathrm{SiN}_{X}$ film as a bottom gate dielectric layer film and a 600-nm-thick a-Si:H film as an active semiconducting layer were deposited consecutively by a plasma-enhanced chemical vapor deposition (PECVD) at $300{ }^{\circ} \mathrm{C}$ in a single vacuum process. The second lithography step was patterned, followed by a selective dry etching process using inductively coupled plasma (ICP) reactive ion etcher at $10{ }^{\circ} \mathrm{C}$ to form the 3-D FIN-shaped structure. Mask \#3: Ohmic contacts are formed by deposition of an additional layer of heavily doped $\mathrm{n}^{+}$-a Si:H of 50-nm thickness by PECVD at $300{ }^{\circ} \mathrm{C}$. The third lithography was used to define TFT island followed by dry-etching of dual layers of $n^{+}-\mathrm{a}-\mathrm{Si}: \mathrm{H}$ and a-Si:H. Mask \#4: A 100-nm thick layer of Cr was deposited by DC sputtering and was then patterned by lithography to form source/drain electrodes followed by wet-etching processes. Afterwards, the $\mathrm{n}^{+}$-a-Si:H layer between the source/drain electrodes was removed using dry etching process. In the next step, 300-nm-thick $\mathrm{SiN}_{X}$ film was deposited as a top-gate dielectric layer by PECVD at $300{ }^{\circ} \mathrm{C}$. Mask \#5: The dielectric layer deposition is followed by the deposition of $100 \mathrm{~nm}$ Cr layer by DC sputtering and patterning by lithography and wet etching processes as top-gate electrode. Later on, the device was passivated with deposition 300-nm-thick $\mathrm{SiN}_{X}$ film by PECVD at $300{ }^{\circ} \mathrm{C}$. Mask \#6: In the final step, the contact vias were patterned by lithography and opened by dry-etching process for electrical probing. After the DG-TFT was prepared, the PVDF transducer was mechanically laminated over the DG-TFT on a glass substrate via low-temperature anisotropic conductive film (ACF) from Teamchem Materials Company. The laminator (Model: TWB-150; Company: Guangzhou KeFu Instrument Co., 
Guangzhou, China) was used to laminate PVDF over ACF, keeping the pressure at more than $0.3 \mathrm{MPa}$ to completely crush the particles of interfacing ACF layer, and the temperature below $75{ }^{\circ} \mathrm{C}$ to retain the electrical properties of PVDF film (Supplementary Figure S3).

\section{Results and Discussion}

Figure 2a shows the IV characteristics of a-Si:H dual-gate TFT with 3-D FIN-shaped channel structure in a self-driven mode, where $V_{T G}=V_{B G}=V_{D S}$. The force generated from mechanical shock or sudden impact lies in the range of more than $10 \mathrm{~N}$, which may produce output voltage peaks with tens of vs. [19,24]. For a highly-sensitive force sensor, a high voltage peak can be generated in a short time and may damage the AFE and cause reliability issue. However, in the case of the PTGTFT, the output signal upon mechanical or electrostatic shocking is high current instead of high voltage. From Figure 2a, we can observe that under self-driven voltage of $50 \mathrm{~V}$, quite high voltage value to mimic the peak voltage received by the device, the output current is around $100 \mu \mathrm{A}$ which can be safely handled by the AFE. In other words, the sensor is capable of bearing high voltages, thereby proving to have wide dynamic range and good reliability. Figure $2 b$ illustrates that the square root of the output current varies with the self-driven voltage in a linear manner in accordance with Equation (4). The self-driven voltage generated from the PVDF transducer from the respiration process is typically around several voltages well above the threshold voltage of the DG-TFT. Figure $2 c$ plots dynamic response of the sensor upon force load of $1 \mathrm{~N}$ at $6.5 \mathrm{~Hz}$. The sensor is able to trace a dynamically-applied force with a rise time of $50 \mathrm{~ms}$, sufficient for respiration monitoring.
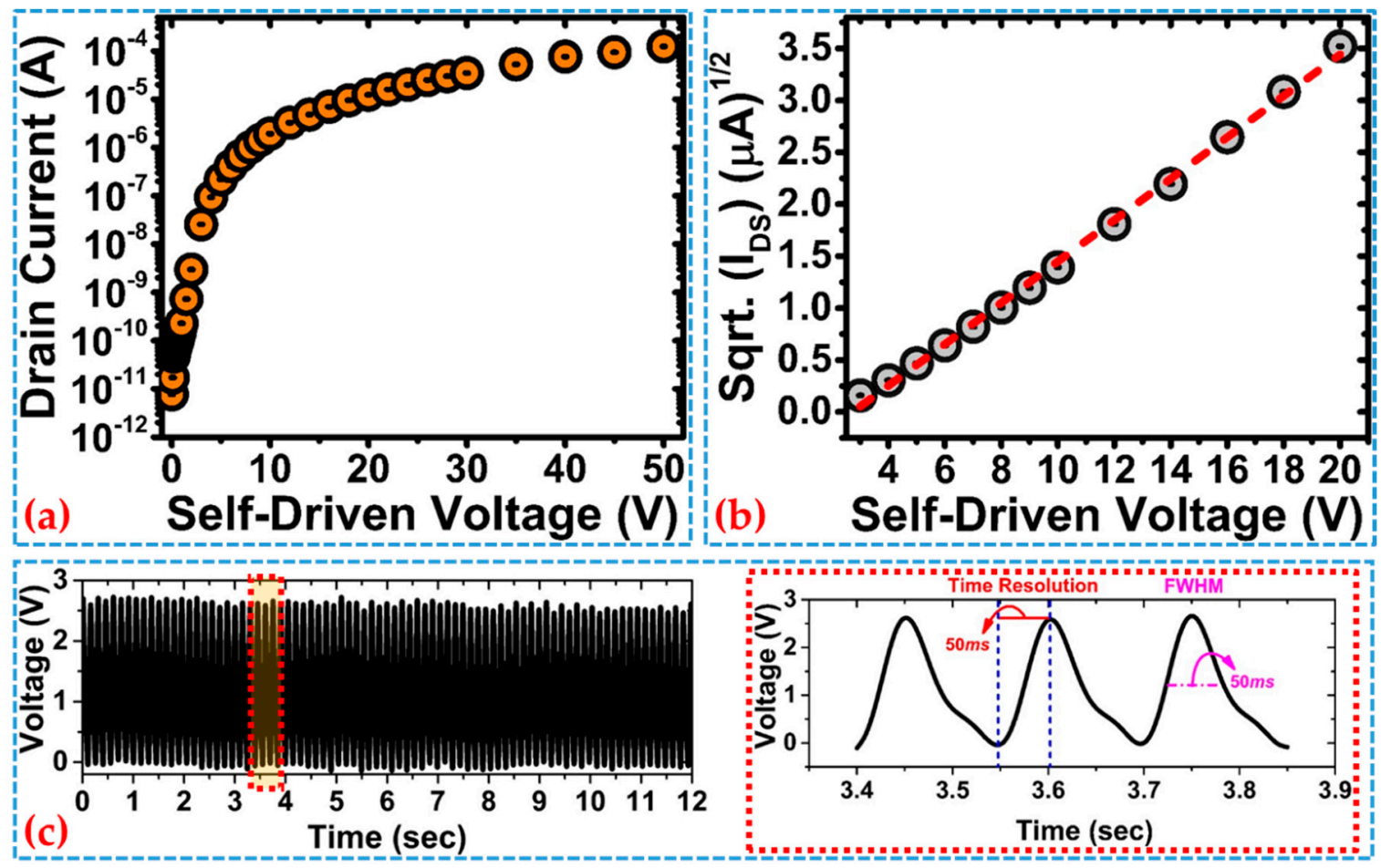

Figure 2. (a) Transfer characteristics of 3-D FIN-shaped DG-TFT, when operating in a self-driven mode; (b) Current variation of the fabricated 3-D FIN-shaped DG-TFT when operating in saturation region; (c) mechanical stability assessment and time-resolution evaluation of PTGTFT sensor at $6.5 \mathrm{~Hz}$ and force of $1 \mathrm{~N}$.

Figure 3a shows the output voltage signals obtained from the AFE of the sensor system. The measurements were performed at the neck and the chest locations when a subject was at rest. Respiration rate was extracted from either the time difference between two peaks or the number of peaks over a specified time interval. The sensor was also collecting respiratory rhythms from multiple locations 
across the body. Figure $3 b$ demonstrates that the signal increased and decreased as the subject exhaled and inhaled, respectively. In the chest location, the rib cage expanded during exhalation, making the PTGTFT experience an elevated force and leading to a signal increase. Contrarily, inhalation contracted the rib cage, driving the PTGTFT to experience a decreased force, thus resulting in the decrease of the output voltage.
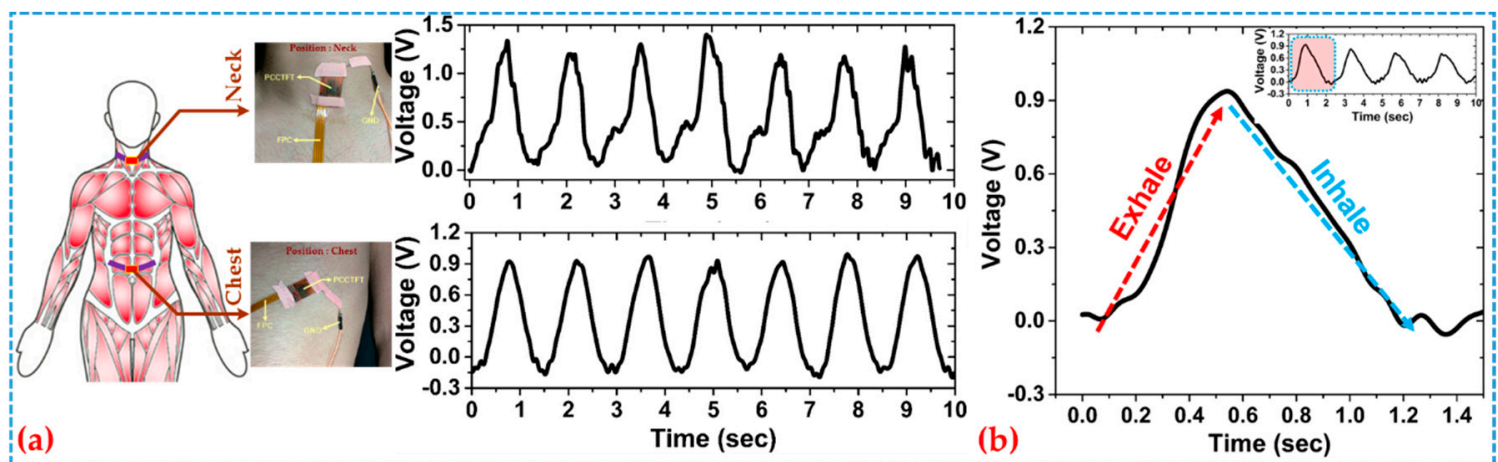

Figure 3. Dynamic response of the sensor at two peripheral points of (a) neck and chest; (b) signal of one complete respiration cycle.

Moreover, Figure 4a illustrates the output voltage signal of a monitored subject for different respiration modes, i.e., apnea, deep, moderate, and rapid. The time dependence of the voltage signals indicates the steady variation in the process of three breathing patterns. Shown in Figure $4 \mathrm{a}$, as well, the zero voltage signal before and after different respiration modes corresponded to the subject holding the breath, thus showing the potential of indication for the diagnosis and warning of apnea. In addition to this, to demonstrate the sensor's ability, the respiration rate of a subject was measured for an extended period of time (over one week), for two different circumstances of normal breathing and moderate breathing. The respiration rate was calculated and averaged for each position and was compared with the respiration derived from the ECG measurement (EDR). The resulted average value from both devices for each day is listed in Supplementary Table S1. The obtained results found no significant difference between PTGTFT and EDR measurements and infers the viability of PTGTFT for monitoring and analysis of respiratory activities of multi-subjects.

Furthermore, a series of experiments were conducted for the subject in various daily activities, to validate the portability of the sensor for real-time respiration monitoring. The respiration was monitored in four different states, i.e., sitting (state I), lying (state II), standing (state III), and walking (state IV). The experiment for state II was designed to evaluate and simulate the breathing process during sleep, while the other cases were designed to evaluate the respiration patterns of the subject in typical daytime activities. Figure $4 \mathrm{~b}$ illustrates the signal variation during different breathing processes of sitting, sleeping, standing, and walking, respectively. The portion of the waveform where no signal was detected, e.g., in state I and state II, shows a clear sign of pause in a breath rhythm (black box). The measured respiration rates for the four states were $21 / \mathrm{min}, 26 / \mathrm{min}, 32 / \mathrm{min}$, and 38/min, which are reasonable respiration rates for the healthy adults [27]. In the case of blank experiment or during breath holding (apnea), no signal occurred, which means that the generated signal corresponds to the physiological parameter. It also indicated that mechanical motion of monitored individual can be acquired by the PTGTFT. Therefore, in the event of coughing, where the air is entrapped in the lungs due to shutting of vocal cords, the rib-cage muscle experiences the substantial force, resulting in the sudden rise of output voltage as shown in Supplementary Figure S5, thus showing the effectiveness of the PTGTFT towards additional monitoring of human motion and activities, e.g., swallowing, sneezing, etc. 

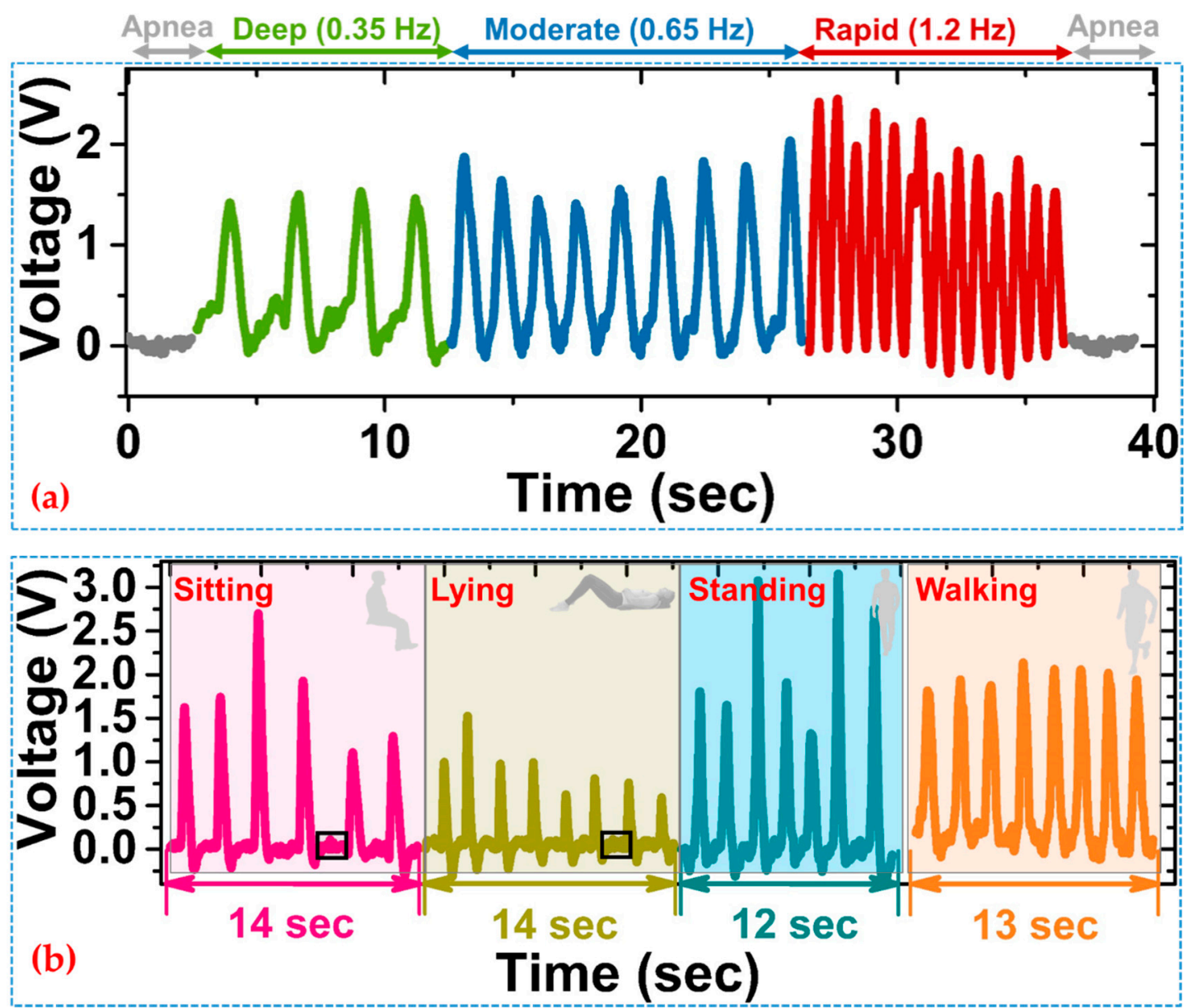

Figure 4. (a) Dynamic response of the sensor in different respiration modes at rest; (b) respiration monitoring tests for three different daily activities when the subject is (I) sitting; (II) lying; (III) standing, and (IV) walking.

To evaluate normality of human breathing patterns, three phases of respiration are examined: Inspiratory, expiratory, and pause phase. Thanks to the quick dynamic response this sensor has, phases of respiration can be accurately monitored. Figure 5 a shows a typical voltage waveform obtained from a series of respiration cycles where the three respiration phases in each respiration cycle are highlighted. The expiratory time $\left(\mathrm{T}_{\mathrm{E}}\right)$ corresponds to the time of signal rise and the inspiratory time $\left(T_{I}\right)$ and pause time $\left(T_{P}\right)$ correspond to the time when the signal decreases and the signal keeps at the minimum. Figure $5 b-d$ presents the relation of $T_{E}$ and $T_{I}+T_{P}$ for each respiration mode of deep, moderate, and rapid breathing for a monitored subject, and the patterns distribute around the lines of $\mathrm{T}_{\mathrm{I}}+\mathrm{T}_{\mathrm{P}}: \mathrm{T}_{\mathrm{E}}=1: 1.5,2: 1.52$, and 3:1.5, respectively. When inhaling and exhaling are successive without being stopped, it means $\mathrm{T}_{\mathrm{P}}$ is much smaller than $\mathrm{T}_{\mathrm{E}}$ and $\mathrm{T}_{\mathrm{I}}$, and the plot moves to the red line of 1:1.5. Similarly, when the subject is experiencing deep breathing, the pause time will increase and the plot will move towards the green line of 3:1.5, which is equal to the usual I:E ratio [6]. Thus, phase analysis of respiration patterns can give a better understanding of human respiratory dynamics. Our study shows that the proposed sensor enables efficient collection of respiration signal waveforms for real-time monitoring that could be utilized during various medical assessments similar to the application of a fiber-optic sensor to study the relationship between anxiety and respiratory rates for the patients undergoing MRI treatment [31]. In such application scenarios, the PTGTFT would be potentially capable of acquiring and analyzing respiratory activities during intermittent stages of assessment as well. 

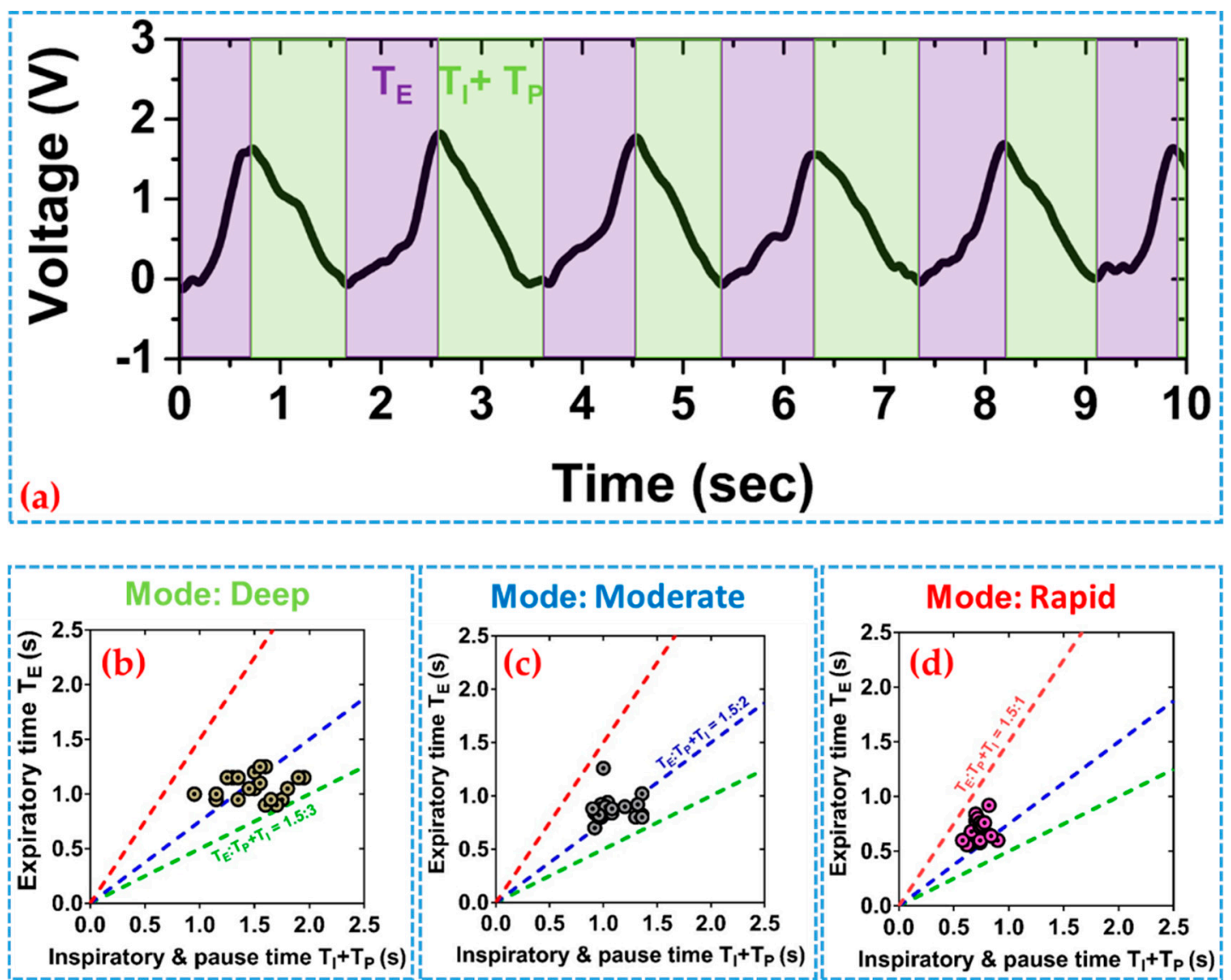

Figure 5. (a) Phase analysis of human respiration rhythm; relation of expiratory time and sum of inspiratory and pause time in during respiration mode of (b) deep, (c) moderate, and (d) rapid.

\section{Conclusions}

An active self-driven sensor for real-time respiration monitoring was proposed and studied. An analytical model was established to elaborate its sensitivity. Such an active sensor differs from passive sensors in that it provides signal rectification and amplification through a piezoelectric-transducer-gated TFT. The highly-sensitive and reliable sensor allows for monitoring respiratory activities at different body locations and analysis of respiration phases in real time.

Supplementary Materials: The following are available online at http://www.mdpi.com/1424-8220/19/14/3241/s1, Figure S1: Summary of recently-developed wearable respiration rate sensors, Figure S2: Schematic illustration of fabrication steps of DG-TFT, Figure S3: schematic illustration of PTGTFT fabrication by the integration of PVDF film and 3-D FIN-shaped DG-TFT, Figure S4: Electrical characterization of 3-D FIN-shaped DG-TFT, Figure S5: Electric response of the PTGTFT to successive mechanical motions; Table S1: Comparison of respiration rate monitoring between PTGTFT and EDR.

Author Contributions: A.R. and K.W. conceived and designed the experiments; A.R., E.I., W.L., Q.Z., Y.X., and H.O. performed the experiments; A.R. and K.W. conducted the data analysis; K.W. supervised and coordinated this study; A.R. and K.W. wrote the paper; all authors read and revised the manuscript.

Funding: This research was supported in part by the 5th Batch Guangdong Innovative and Entrepreneurial Research Team Program under Contract 2014ZT05D340 and in part by the Guangzhou City under Contract 201508020121.

Conflicts of Interest: The authors declare no conflict of interest. 


\section{References}

1. Benjamin, E.J.; Muntner, P.; Alonso, A.; Bittencourt, M.S.; Callaway, C.W.; Carson, A.P.; Chamberlain, A.M.; Chang, A.R.; Cheng, S.; Das, S.R.; et al. Heart disease and stroke statistics-2019 update: A report from the American heart association. Circulation 2019, 139, e56-e66. [CrossRef] [PubMed]

2. Cretikos, M.A.; Bellomo, R.; Hillman, K.; Chen, J.; Finfer, S.; Flabouris, A. Respiratory rate: The neglected vital sign. Med. J. Aust. 2008, 188, 657-659. [CrossRef] [PubMed]

3. Zhao, Z.; Yan, C.; Liu, Z.; Fu, X.; Peng, L.M.; Hu, Y.; Zheng, Z. Machine-Washable Textile Triboelectric Nanogenerators for Effective Human Respiratory Monitoring through Loom Weaving of Metallic Yarns. Adv. Mater. 2016, 28, 10267-10274. [CrossRef] [PubMed]

4. Lochner, C.M.; Khan, Y.; Pierre, A.; Arias, A.C. All-organic Optoelectronic Sensor for Pulse Oximetry. Nat. Commun. 2014, 5, 5745. [CrossRef] [PubMed]

5. Chen, L.Y.; Tee, B.C.K.; Chortos, A.L.; Schwartz, G.; Tse, V.; Lipomi, D.J.; Wong, H.S.P.; McConnell, M.V.; Bao, Z. Continuous Wireless Pressure Monitoring and Mapping with Ultra-small Passive Sensors for Health Monitoring and Critical Care. Nat. Commun. 2014, 5, 5028. [CrossRef] [PubMed]

6. Tatara, T.; Tsuzaki, K. An apnea monitor using a rapid-response hygrometer. J. Clin. Monit. Comput. 1997, 13, 5-9. [CrossRef]

7. Massaroni, C.; Nicolò, A.; Lo Presti, D.; Sacchetti, M.; Silvestri, S.; Schena, E. Contact-Based Methods for Measuring Respiratory Rate. Sensors 2019, 19, 908. [CrossRef]

8. Oki, Y.; Kaneko, M.; Fujimoto, Y.; Sakai, H.; Misu, S.; Mitani, Y.; Yamaguchi, T.; Yasuda, H.; Ishikawa, A. Usefulness of the 6-minute walk test as a screening test for pulmonary arterial enlargement in COPD. Int. J. Chronic Obstr. Pulm. Dis. 2016, 11, 2869-2875. [CrossRef]

9. Lu, K.; Yang, L.; Seoane, F.; Abtahi, F.; Forsman, M.; Lindecrantz, K. Fusion of Heart Rate, Respiration and Motion Measurements from a Wearable Sensor System to Enhance Energy Expenditure Estimation. Sensors 2018, 18, 3092. [CrossRef]

10. Chon, K.H.; Dash, S.; Kihwan, J. Estimation of Respiratory Rate from Photoplethysmogram Data Using Time-Frequency Spectral Estimation. IEEE Trans. Biomed. Eng. 2009, 56, 2054-2063. [CrossRef]

11. Zhang, X.; Ding, Q. Respiratory rate monitoring from the photoplethysmogram via sparse signal reconstruction. Physiol. Meas. 2016, 37, 1105-1119. [CrossRef] [PubMed]

12. Kim, H.; Kim, J.Y.; Im, C.H. Fast and robust real-time estimation of respiratory rate from photoplethysmography. Sensors 2016, 16, 1494. [CrossRef] [PubMed]

13. Dieffenderfer, J.; Goodell, H.; Mills, S.; McKnight, M.; Yao, S.; Lin, F.; Beppler, E.; Bent, B.; Lee, B.; Misra, V.; et al. Low-Power Wearable Systems for Continuous Monitoring of Environment and Health for Chronic Respiratory Disease. IEEE J. Biomed. Health Inform. 2016, 20, 1251-1264. [CrossRef] [PubMed]

14. Guder, F.; Ainla, A.; Redston, J.; Mosadegh, B.; Glavan, A.; Martin, T.J.; Whitesides, G.M. Paper-based electrical respiration sensor. Angew. Chem. Int. Ed. 2016, 55, 5727-5732. [CrossRef] [PubMed]

15. Pang, Y.; Jian, J.; Tu, T.; Yang, Z.; Ling, J.; Li, Y.; Wang, X.; Qiao, Y.; Tian, H.; Yang, Y.; et al. Wearable humidity sensor based on porous graphene network for respiration monitoring. Biosens. Bioelectron. 2018, 116, 123-129. [CrossRef]

16. Kano, S.; Dobashi, Y.; Fujii, M. Silica Nanoparticle-Based Portable Respiration Sensor for Analysis of Respiration Rate, Pattern, and Phase during Exercise. IEEE Sens. Lett. 2018, 2, 1-4. [CrossRef]

17. Yamada, T.; Hayamizu, Y.; Yamamoto, Y.; Yomogida, Y.; Izadi Najafabadi, A.; Futaba, D.N.; Hata, K. A Stretchable Carbon Nanotube Strain sensor for Human-Motion Detection. Nat. Nanotechnol. 2011, 6, $296-301$. [CrossRef]

18. Gong, S.; Schwalb, W.; Wang, Y.; Chen, Y.; Tang, Y.; Si, J.; Shirinzadeh, B.; Cheng, W. A Wearable and Highly Sensitive Pressure Sensor with Ultrathin Gold Nanowires. Nat. Commun. 2014, 5, 3132. [CrossRef]

19. Amjadi, M.; Kyung, K.U.; Park, I.; Sitti, M. Stretchable, Skin Mountable, and Wearable Strain Sensors and Their Potential Applications: A Review. Adv. Funct. Mater. 2016, 26, 1678-1698. [CrossRef]

20. Hamdani, S.T.A.; Anura, F. The application of a piezo-resistive cardiorespiratory sensor system in an automobile safety belt. Sensors 2015, 15, 7742-7753. [CrossRef]

21. Lei, K.F.; Hsieh, Y.Z.; Chiu, Y.Y.; Wu, M.H. The structure design of piezoelectric poly (vinylidene fluoride) (PVDF) polymer-based sensor patch for the respiration monitoring under dynamic walking conditions. Sensors 2015, 15, 18801-18812. [CrossRef] [PubMed] 
22. Zang, Y.; Zhang, F.; Huang, D.; Gao, X.; Di, C.A.; Zhu, D. Flexible Suspended Gate Organic Thin-Film Transistors for Ultra Sensitive Pressure Detection. Nat. Commun. 2015, 6, 6269. [CrossRef] [PubMed]

23. Nie, B.; Li, R.; Cao, J.; Brandt, J.D.; Pan, T. Flexible Transparent Iontronic Film for Interfacial Capacitive Pressure Sensing. Adv. Mater. 2015, 27, 6055-6062. [CrossRef] [PubMed]

24. Xue, H.; Yang, Q.; Wang, D.; Luo, W.; Wang, W.; Lin, M.; Liang, D.; Luo, Q. A wearable pyroelectric nanogenerator and self-powered breathing sensor. Nano Energy 2017, 38, 147-154. [CrossRef]

25. Dinh, T.; Phan, H.P.; Nguyen, T.K.; Qamar, A.; Woodfield, P.; Zhu, Y.; Nguyen, N.T.; Dao, D.V. Solvent-free fabrication of biodegradable hot-film flow sensor for noninvasive respiratory monitoring. J. Phys. D. Appl. Phys. 2017, 50, 215401. [CrossRef]

26. Chen, S.W.; Wu, N.; Ma, L.; Lin, S.Z.; Yuan, F.; Xu, Z.S.; Li, W.B.; Wang, B.; Zhou, J. Noncontact heartbeat and respiration monitoring based on a hollow microstructured self-powered pressure sensor. ACS Appl. Mater. Interfaces 2018, 10, 3660-3667. [CrossRef] [PubMed]

27. Zhang, H.; Zhang, J.; Hu, Z.; Quan, L.; Shi, L.; Chen, J.; Xuan, W.; Zhang, Z.; Dong, S.; Luo, J. Waist-wearable wireless respiration sensor based on triboelectric effect. Nano Energy 2019, 59, 75-83. [CrossRef]

28. Rasheed, A.; Iranmanesh, E.; Li, W.; Feng, X.; Ou, H.; Wang, K. A Wearable Self-Driven Impulse Sensor Based on a Mechanical-Field-Coupled Thin-Film Transistor. IEEE Electron Device Lett. 2018, 39, 1756-1759. [CrossRef]

29. Rasheed, A.; Iranmanesh, E.; Li, W.; Ou, H.; Andrenko, A.S.; Wang, K. A wearable autonomous heart rate sensor based on piezoelectric-charge-gated thin-film transistor for continuous multi-point monitoring. In Proceedings of the 39th Annual International Conference of the IEEE, Engineering in Medicine and Biology Society (EMBC), Jeju, Korea, 11-15 July 2017; pp. 3281-3284. [CrossRef]

30. Li, W.; Rasheed, A.; Feng, X.; Iranmanesh, E.; Wang, K.; Ou, H.; Chen, J.; Deng, S.; Xu, N. Mechanical-field-coupled thin-film transistor for tactile sensing with $\mathrm{mN}$ dynamic force detection capability and wearable self-driven heart rate monitoring with $\mu \mathrm{W}$ power consumption. In Proceedings of the 2017 IEEE International Electron Devices Meeting (IEDM), San Francisco, CA, USA, 2-6 December 2017; pp. 18.3.1-18.3.4. [CrossRef]

31. Dziuda, L.; Zielińsk, P.; Baran, P.; Krej, M.; Kopka, L. A study of the relationship between the level of anxiety declared by MRI patients in the STAI questionnaire and their respiratory rate acquired by a fiber-optic sensor system. Sci. Rep. 2019, 9, 4341. [CrossRef]

(C) 2019 by the authors. Licensee MDPI, Basel, Switzerland. This article is an open access article distributed under the terms and conditions of the Creative Commons Attribution (CC BY) license (http://creativecommons.org/licenses/by/4.0/). 